\title{
Design Channel Nonpersistent CSMA MAC Protocol Model for Complex Wireless Systems Based on SoC Methodology
}

\author{
Ibrahim A. Aref, Tarek A. El-Mihoub and Khadiga A. Ben Musa \\ Computer Engineering Department University of Tripoli Tripoli, Libya \\ \{iaref \& tmihoub\}@tripoliuniv.edu.ly, k.benmusa@ec.uot.edu.ly
}

\begin{abstract}
This paper presents Carrier Sense Multiple Access (CSMA) communication model based on SoC design methodology. Such model can be used to support the modelling of the complex wireless communication systems; therefore the use of such communication model is an important technique in the construction of high performance communication. SystemC has been chosen because it provides a homogeneous design flow for complex designs (i.e. SoC and IP based design). We use a swarm system to validate CSMA designed model and to show how advantages of incorporating communication early in the design process. The wireless communication created through the modelling of CSMA protocol that can be used to achieve communication between all the agents and to coordinate access to the shared medium (channel).
\end{abstract}

Index Terms - SystemC, Modelling, Simulation, CSMA

\section{Introduction}

The communication modelling of the wireless complex communication system becomes a big challenging since the complexity of modelling and designing such systems are increased. As a result, the system designers are moving towards use System-on-Chip (SoC) methodologies which are a complex integrated circuit that integrates the major functional elements of a large system into a single chip. It may integrate different types of components such as digital elements, electronic elements or non-electrical parts (sensors) as well as software components [5, 7]. Those designing methodologies can be used as powerful modelling and simulation techniques to design, model and verify the complex wireless systems as well as to address all aspects consistently and efficiently. SystemC [10] is emerging as a suitable design and modelling language, because it provides a consistent methodology for the design and refinement of complex digital systems [11]. This methodology is essential to manage complexity and enhance designer productivity. It allows the designer to view designs at different levels of abstraction and in particular advocates the evaluation of the system performance early in the design cycle, and its use in guiding the refinement process into lower levels of abstraction [7]. It is desirable to apply the same design methodology for complete complex systems, including any off-chip components.

The modelling and designing of complex wireless communication systems is complicated and challenging, because during system design exploration, system design options may affect communication behavior, and communication design options may impact system design [14].
For this reason, it is important to introduce the integration of communication modelling into the design modelling at an early stage of system development. Moreover, by using SystemC as a design tool, HW, SW and communication can be modeled, refined and validated together; the result is a twodimensional design space as shown in Fig. 1. The vertical dimension addresses both the refinement of the system model and the optimization of its algorithms; during this process the communication model is used both to drive architectural exploration and to verify that communication requirements are met. The horizontal dimension represents the design space of the communication model in which different topologies and parameters can be verified $[1,4]$.

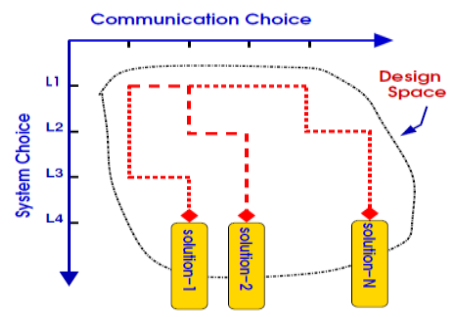

Fig. 1 Introduce Communication Modelling into Design Modelling

In this paper, an intelligent engineering swarm system $[12,13]$ is selected as a demonstration in order to validate our CSMA communication model. Swarm intelligence is the discipline that deals with natural and artificial systems composed of many individuals that coordinate using decentralized control and self-organization [12,13]. It is a very complex system modeled based on the SoC design methodology and partitioned along different parameters. The whole modelled system is introduced to simulate the behavior of the agents (mobile units) that form a mobile ad-hoc communication network. The wireless communication between agents is addressed based on nonpersistent CSMA MAC protocol. This protocol is designed to handle access to the shared channel [6]. In this work, CSMA is employed to accomplish communication between the agents. It is classified as a random access protocol and was developed to minimize the chance of collision and therefore increase the system performance. It uses a contention-based approach to channel access, and does not require time synchronization $[9,15]$. The rest of the paper is organized as follows: the next section 
presents how to use SystemC methodology to create CSMA model, Section 3 presents communications scenarios based on MAC protocols. The simulation platform will be explained in Section 4 and then Section 5 presents the results and discussion. Finally, conclusions are drawn in Section 6.

\section{Using SystemC Methodology to Create CSMA Model}

SystemC design methodology provides a consistent framework for the design and modelling of complex systems at different levels of abstraction. This is important to design complex systems (SoC), where it is necessary to determine the system performance before a prototype is constructed, in order to evaluate the merit of different implementations. SystemC can describes hardware at high levels of abstraction, the primary advantage to designing any system at the system level is having the ability to implement that system from the highest level of abstraction, i.e specifications model. From a system design methodology perspective $[3,8]$, there are a number of intermediate models that can be defined and then used to reduce the complexity of the system design process. Using intermediate models divides the entire design into slices (small design tasks). Each task is represented by a model and has a specific design objective. Thus, each model can be simulated and the results independently validated [7].

\section{Communications Scenarios based on MACs}

To meet the requirements of designing an efficient communication system, multiple access communication (MAC) protocols are necessary to allow multiple resources to share transmission medium simultaneously, which means these multiple access schemes employed to manage multiple access issues based on a multiplexing technique. Moreover, these schemes determine how data streams can be read efficiently from each communication node and then transmitting over a shared medium and finally broadcast to all the network nodes [6]. In MAC Protocols, the assumption made that all communication occurs on single channel on which all stations can transmit and receive and no separate signaling channel. There are two basic approaches, the first one is Scheduling, e.g. TDMA, Reservation, Polling, Token Passing. The advantage of this approach is that ready stations transmit and stations are always guaranteed to get through on the predefined schedule. The disadvantage of it is that wastes time and bandwidth. The other approach is random access, e.g. Carrier sensing (If carrier sensing, stations can tell if channel is in use before trying to transmit), no carrier sensing (transmit any time, detect collisions by absence of ACK. e.g, slotted ALOHA, ALOHA). The main type of carrier sense is Carrier sense multiple access - CSMA (sense if channel is busy, send if idle ,collisions if two or more stations send at same time), CSMA/CD: with Collision Detection(sense if channel is busy or a collision occurs, stops sending if a collision is noticed.eg, IEEE 802.3)[6, 2]. The advantage of this method is that ready stations transmit anytime. The disadvantage is that stations not guaranteed to get through (collisions may continue to occur). There are three types of CSMA: Nonpersistent CSMA (wait entire back-off period before sensing channel again high efficiency, high delay), p-persistent CSMA wait until channel idle, then transmit with probability p spread out transmission attempts by stations that have been waiting, delay and efficiency can be balanced), 1-persistent CSMA (start transmission as soon as channel becomes idle low delay, low efficiency) [9, 15].

In this work, we illustrate how CSMA technique is used to construct communication between the agents in intelligent engineering swarm system based on a shared medium. It is a contention based protocols. We need to show here that our target not to investigate the protocol issues but we need to prove that incorporating the wireless features early in the design methodology is very advantageous. Also we need to show how to integrate communication modelling and the design modelling early in the system development and then how to allow us to investigate the system very easily and make changes quickly. In the next sections, The CSMA models are carried out at two level of abstraction, the first one at high abstraction level, which means the communication between the agents is modelled based on a shared variable (primitive channel) such FIFO. The other one is refine communication by inserting wireless communication channel model.

\section{A. Non-persistent CSMA Modelling at High Abstraction Level}

The CSMA protocol is a network arbitration protocol which regulates communication between several resources who communicate by a unique channel. It is a widely studied protocol using various techniques [6]. This part of the work is focusing on non-persistent CSMA technique because of the simplicity and the good performance. We assume here that the agents are not limited by transmit power, which means each agent can transmit at any power required to reach all the other agents in the system (fully connected topology). The swarm system model consists of $\mathrm{N}$ agents distributed uniformly and communicated over shared channel model. As shown in Fig. 2, the nonpersistent CSMA is modeled in SystemC by using two main elements, the mutual exclusion object Mutex and $s c$ signal resolved [3]. The first one lets agents to share shared wireless channel model without colliding by allow for just one agent to send data packets and the other one allows the agents as multiple writers to access wireless channel. An agent that has a packet to send senses the channel by checking the Mutex. If the channel is idle, the agent sends immediately. If the channel is busy, the agent waits a random amount of time and then senses the channel again, but in this case, the packet is rescheduled with the new value of the current position [15].

\section{B. Refine CSMA Model}

To optimize system stability in terms of communication, the system is refined by inserting the wireless channel model, so the stability and reliability of the system can be investigated in order to attain the best performance under the communication effects. Fig. 3 illustrates the system diagram after inserting the wireless channel. The main advantage of inserting a wireless channel model into the system is that it allows us to simulate wireless features, i.e., evaluating the 
effects of inserting noise through the channel. Moreover, we can determine the effect of the communication latency and communication BW to maintain system stability. The main objective is to prove that we can successfully use SystemC methodology with a shared channel model to design a complex wireless communication system such as swarm system.

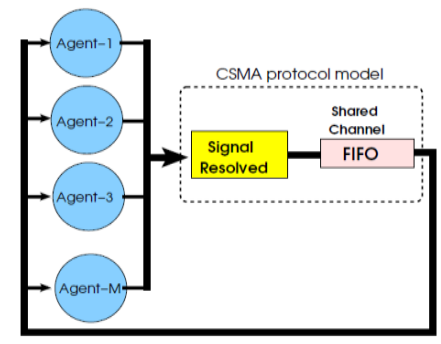

Fig. 2 CSMA Model at High Abstraction Level

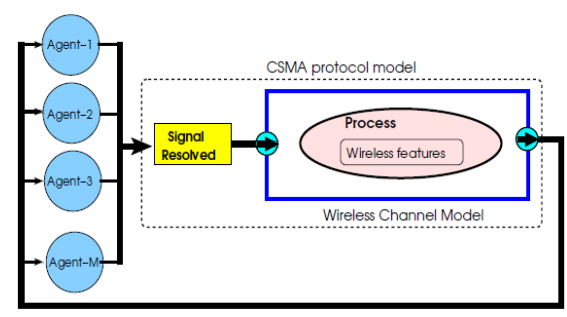

Fig. 3 Refine CSMA Model

\section{Simulation Platform}

In SystemC, functional verification of the modelled system is done through simulation. This process consists of applying a stimulus to the Device Under Test (DUT) and verifying the response against an expected result. At each time step in the simulation, the processes in Fig. 4 are applied to all agents simultaneously, and the positions and velocities of all agents at the next time step are updated accordingly. Subsequently, each agent must send its updated position to the other agents located within their radius of perception.

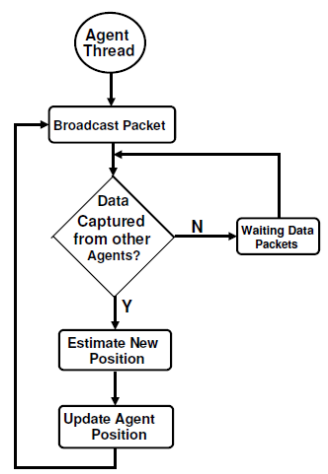

Fig. 4 Data Flow within the Agent Model

In the beginning of the simulation, we assume the agents are distributed uniformly in the space with a defined shape. The shape is created based-on relative position value that is given to each agent, the distance between the relative positions represent the threshold value (Th) that should be maintained by each agent in order to make the system converge. So at the final position, the system can be reached the convergence point if each agent maintains the threshold distance with the agents that located at is radius of perception. Moreover, we need to select one of the agents as leader. If the leader is moved, all the agents should follow, otherwise (leader fixed) all the agent should kept close to each other without colliding and converge around leader based on the relative positions. In this work, $P_{0}$ is selected as a leader. Also there are some initial conditions have to consider in this system such as:

*System data rate that expressed as a number of packets per simulation step.

*PD controller parameters $\left(\mathrm{K}_{\mathrm{p}}\right.$ and $\left.\mathrm{K}_{\mathrm{d}}\right)$, which affect the way agents will interact with each other.

*The size of swarm system; to simplify the simulation, this system has investigated with ten agents $(n=10)$.

*The acceleration and speed of the agents that are bounded based on the error value.

The equations of motion employed in this simulation are symmetrical because all the agents are identical. The simulation program affects the agents movement by modifying only their acceleration; their velocity and position will be updated based on the acceleration value. The simulation takes place on a two-dimensional axis (2D). Each agent has corresponding equations for movement on both $\mathrm{X}$ and $\mathrm{Y}$ axes. The positions, velocities and accelerations of the agents are all $2 \mathrm{D}$ vectors $(\mathrm{x}, \mathrm{y})$.

\section{Experimental Results}

In order to simplify the simulation, only ten agents $(\mathrm{n}=$ 10) are created to run experiments and the relative positions of these agents are defined as mesh. The packet length is fixed (16 bytes). The leader position was located in the corner. Moreover, different scenarios have been simulated with the developed methodology, which means in some cases the leader was fixed and in other cases it was assumed to be movable. All agents started moving with $\left(\mathrm{V}_{\mathrm{x}}\right)$ and $\left(\mathrm{V}_{\mathrm{y}}\right)$ based on the error value. These initial values were provided to the system by the stimulus. All the agents began moving from position $(0,0)$ and distributed themselves around the leader (if the leader was fixed) in a uniform shape based on the initial values of their relative positions. If the leader was movable, the agents followed the leader. All these changes were made in order to validate the developed methodology and then prove that incorporating wireless communication at an early stage of the design flow is very advantageous. Multiple simulations were run to check system behavior and to optimize the model parameters, including simulation steps, transmission rate, communication delay, speed and acceleration of the agents. Another parameter to evaluate the performance is the changing of the relative distance value error $(\varepsilon)$ which is difference between current distance between two agents $\left(\mathrm{P}_{\mathrm{i}}\right)$ and $\left(\mathrm{P}_{\mathrm{j}}\right)$ and equivalent relative position distance $(\mathrm{Th})$ that defined at the beginning of the simulation.

In the second part of the simulation, the agents are 
communicated based on non-persistent CSMA scheme. The experiment is done under the same conditions that illustrated above in the system parameters as indicated in Table(I). The agents aim to converge to the desired agents relative positions defined at the beginning of the simulation. At the end of simulation, the system converged and the simulation was stopped when the total error value was $(\varepsilon<0.01)$. The system behavior (Fig. 5) clearly shows that the agents remained in the same position structure (mesh) throughout the simulations. Thus it is proven that agents consistently and effectively avoid contact with one another. Fig. 6 illustrates the system dynamics curve and Fig. 7 illustrates the converging point of the system, which was obtained at 1340 simulation steps.

TABLE I System Parameters

\begin{tabular}{|c|c|}
\hline Parameters & Values \\
\hline Error $(\varepsilon)$ & 0.01 \\
\hline Data rate & 1 Packet/simulation step \\
\hline Range of the speed & -1.0 to 1.0 \\
\hline Range of acceleration & -0.2 to 0.2 \\
\hline Kp and Kd & $0.03 \& 0.1$ \\
\hline
\end{tabular}

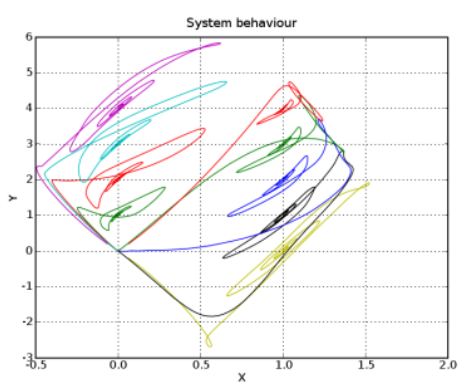

Fig. 5 System Behaviour

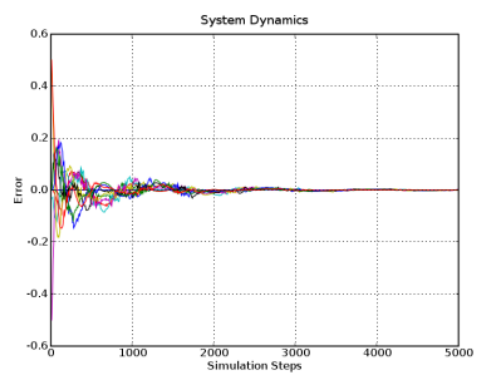

Fig. 6 System Dynamics

\section{Conclusions}

In this work, the conventional SystemC design methodology is used to model nonpersistent CSMA MAC protocol. The design exploration space is changed into two dimensions: system (HW/SW) that is addressed exploration and refinement of the system and the other dimension is communication that addressed wireless features. For instance, the CSMA model becomes a part of the SystemC methodology and then can be inserted into any system. Also we can also introduce some communication delays, communication protocols and noise. Modelling CSMA protocols with SystemC allowed us to build up a software component that is ready-to-used in wireless development prototyping. This software component, which is CSMA model, could be conveniently being used as a substitution of hardware and consequently to cost reduction. There are some non-persistent CSMA protocols had been model before, but the ways and purposes of modelling these protocols are unlike ours, because our aim here is not to investigate these protocol but to prove that inserting wireless communication features into the SoC design methodology are very advantages. In the last stage of this work, the CSMA communication model is validated by modelling of a swarm system that selected as a case study. The final results of the modelled system have been validated and it has been proving that communication has a big impact in system dynamics, i.e., small changes in the wireless specifications create big changes in the system dynamics.

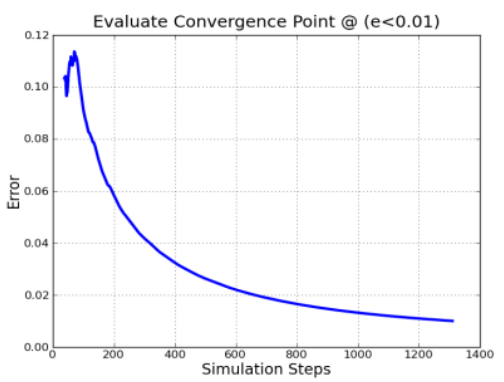

Fig. 7 Convergence Point at 0.01

\section{References}

[1] I. Aref. Wireless Extension to the Existing SystemC Methodology. PhD thesis, School of Eng., University of Glasgow, Glasgow, UK, Feb. 2011.

[2] H. Bidgoli. Handbook of Computer Networks. J. Wiley and Sons, 2008.

[3] D. Black and J. Donovan. SystemC: From the Ground-up. Kluwer Academic Publishers, first edition, 2004.

[4] N. Bombieri, F. Fummi, and D. Quaglia. System/network design-space exploration based on tlm for networked embedded systems. ACM Transactions on Embedded Computing Systems, 9(4), Mar. 2010.

[5] R. Domer, D. Gajski, and A. Gerstlauer. Specc methodology for highlevel modelling. IEEE Electronic Design Processes Workshop EDP 2002.

[6] B. A. Forouzan. Data Communications and Networking. McGraw-Hill Science/Engineering/Math, forth edition, 2006.

[7] D. Gajski, J. Zhu, R. Domer, A. Gerstlauer, and S. Zhao. Spec C: Specification Language and Methodology. 2000.

[8] A. Ghosh, S. Tjiang, and R. Chandra. System modelling with systemc. In ASIC, 2001.

[9] S. Glisic and P. Leppänen. Wireless Communications: TDMA versus CDMA. Kluwer Academic Publishers, 1997.

[10] IEEE_Computer_Society. Ieee standard SystemC language reference manual. IEEE Std 1666-2005, pages 1-423, Mar. 2006.

[11] OSCI. SystemC User's Guide. Open SystemC Initiative, 2002.

[12] H. Parunak. Engineering swarming systems. Kluwer, 2004.

[13] S. Chen and H. Fang. Modelling and control of scalable engineering swarm. The 6th World Congress on Intelligent Control and Automation, June 2006.

[14] F.Stefanni, D.Quaglia and F. Fummi. SystemC simulation of networked embedded systems. Springer Netherlands, 36:201-211, May 2009.

[15] W. Yue and Y. Matsumoto. Performance Analysis of Multi-Channel and Multi-Traffic on Wireless Communication Networks. Kluwer Academic Publishers, first edition, 2002. 